\title{
HIGH-PRESSURE STUDIES OF SEMICONDUCTORS IN THE FAR-INFRARED: DONOR STATES IN QUASI-2D
}

\author{
B.A. Weinstein ${ }^{a}$, J.G. TischleR ${ }^{a}$, R.J. Chen ${ }^{b}$, H.A. Nickel ${ }^{a}$, \\ B.D. $\mathrm{McCombe}^{a}$, A.B. Dzyubenko ${ }^{c}$ and A. SivaChenko ${ }^{d}$ \\ ${ }^{a}$ Department of Physics, SUNY Buffalo, NY, 14260-1500, USA \\ ${ }^{b}$ Brimrose Corporation of America, Baltimore, Md., 21236, USA \\ ${ }^{c}$ General Physics Institute, RAS, Moscow, Russia \\ ${ }^{d}$ Weizman Institute, Rehovot, Israel
}

We review recent experimental advances by the Buffalo group in performing far-infrared magnetospectroscopy under fine tuning of applied high hydrostatic pressure. Experiments are reported for the effects of pressure on $\mathrm{Si}$ donors in modulation doped GaAs/AlGaAs quantum wells. We clearly observe pressure-mediated competition between free (i.e., Landau level) and bound electron states - the latter arising from both neutral $\left(D^{0}\right)$ and charged $\left(D^{-}\right)$donor species. With increasing pressure, there is a progression of the observed spectra from being dominated by cyclotron resonance and the $D^{-}$singlet (or singlet-like bound magnetoplasmon) transitions, to showing the $D^{0} 1 s \rightarrow 2 p^{+}$line. The main reason for this evolution is the decrease in electrons due to the crossover of the Si levels associated with the $\Gamma$ (well) and $X$ (barrier) conduction minima. Indeed, for pressures above $30 \mathrm{kbar}$ the $\Gamma$ (well) $-X$ (barrier) crossover quenches all the transitions. However, we find strong evidence that electrons are independently lost to a trap, which becomes active several kbar below this crossing. A possible candidate for this trap is residual Se impurities in the barriers. We present the results of detailed numerical calculation which are found to agree very well with the measured field dependencies of the cyclotron resonance, $D^{0}$ and $D^{-}$transition energies. In the sample with the highest doping, a new transition is observed for fields and pressures above 7.5 $\mathrm{T}$ and 5 kbar. Reasons for this apparent anomaly are discussed.

PACS numbers: 73.20.Hb, 73.20.Dx, 78.66.Fd 


\section{Introduction}

Many fundamental issues concerning small-energy excitations in semiconductors, e.g., those associated with impurity states, excitons, plasmons, spin and exchange coupling, quantum Hall effect, etc., can benefit from advances in methodsfor performing far infrared (FIR) magnetospectroscopy under in-situ-tunable high hydrostatic pressures. By high, we mean outside the practical range $(\approx 15 \mathrm{kbar})$ of gas and oil bombs. In-situ-tunable is emphasized here because of the small energy of the excitations (often $\approx$ a few $\mathrm{meV}$ ) and the rapid pressure-shift of the conduction band extrema $(\approx 5-10 \mathrm{meV} / \mathrm{kbar})[1]$. These two facts of nature conspire against employing standard clamp diamond-anvil cells (DACs), in which it is extremely difficult to achieve pressure resolution and repeatability of less than a few kbar. Since clamp DACs are convenient for inserting into the restricted geometry of cryogenic FIR magnetooptical experiments, they have often been used for such studies in the past [2-5]. However, without in situ tuning, it becomes largely a matter of luck to detect the many fascinating resonant and anticrossing interactions that are possible in semiconductors. This has been the motivation for the recent instrumentation advances by the SUNY at Buffalo group [6].

The principal difficulties in designing a convenient pressure-tunable DAC for cryogenic FIR magnetospectroscopy derive from four criteria. The apparatus should (i) elude substantial heat losses, (ii) maintain unblocked FIR access, (iii) allow easy visible-light access for ruby pressure-calibration, and (iv) avoid disturbing the field - requiring nonmagnetic materials with lower strength. These criteria figure prominently in deciding on the best type of force generating mechanism to drive the DAC. In the present design, we choose not to use mechanical linkages to a force generating device outside the cryostat $[7,8]$. Rather, we elect to provide the force via gas-pressurized bellows located inside the cryostat. This allows greater flexibility in meeting design features (ii)-(iv), while presenting no added drawbacks for managing heat loss. Although ${ }^{4} \mathrm{He}$ is certainly the best medium to pressurize the bellows, its freezing pressure of $13.8 \mathrm{MPa}$ at $4.2 \mathrm{~K}$ leads to a major limitation on the force available in small magnet-bore cryostats using standard single bellows designs $[9,10]$. Assuming reasonable diamond tip sizes $(\approx 0.4-0.7 \mathrm{~mm})$ for FIR spectroscopy, a force of up to $10 \mathrm{kN}$ is required to achieve DAC pressures above $30 \mathrm{GPa}$. However, in order to generate $10 \mathrm{kN}$ at $4.2 \mathrm{~K}$, the bellows diameter must be at least $38 \mathrm{~mm}$. Allowing for the harness holding the bellows, vacuum can, etc., this generally would require a magnet-bore exceeding $50 \mathrm{~mm}$ - a substantial expense if high fields exceeding $10 \mathrm{~T}$ are desired. The severity of this limitation is more apparent if one considers that essentially zero pressure is produced in the DAC unless one exceeds the initial gasket indentation force which, of necessity, must be applied to the DAC gasket during the sample loading procedure. These considerations have led us to develop the stacked double-bellows ram [11] described below, which enables the maximum bellows force to be doubled without increasing the geometrical diameter.

\section{Double-bellows ram and FIR DAC}

The design details of the present FIR DAC apparatus, including an expanded components-drawing of the stacked double-bellows ram, are shown in Figs. 1a 
and $b$ [6]. The principal components of this apparatus are fabricated from stainless steel, except the DAC itself (parts 18-25) made from hardened $\mathrm{BeCu}$ alloy, the copper paraboloidal FIR focusing cones (15 and 26), the quartz optical fiber (12), and the Ga-doped Ge FIR detector (11). All of the materials used are suitable for high magnetic field applications.
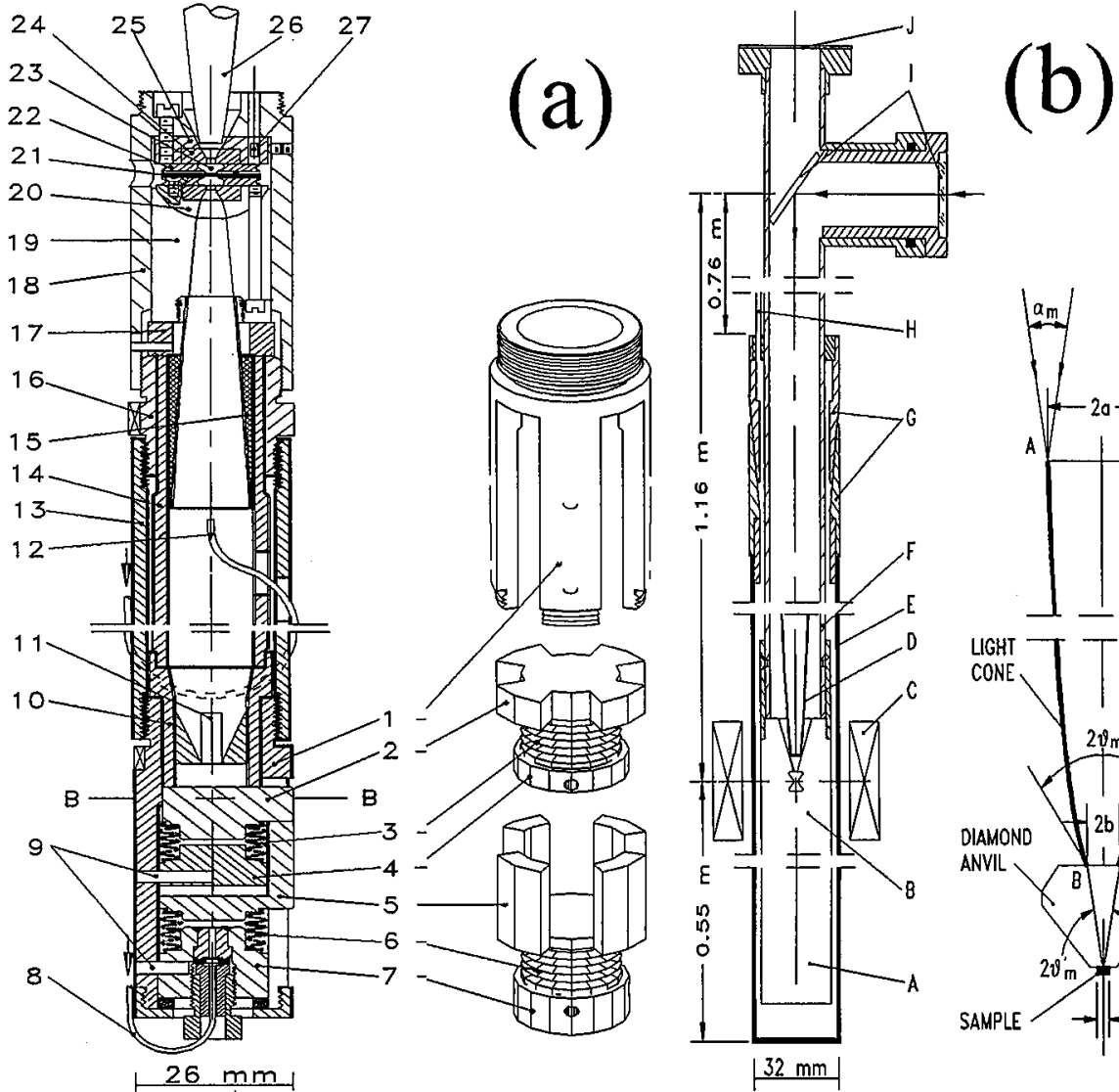

Fig. 1. Design details of the tunable high-pressure FIR-DAC apparatus. (a) Assembly drawing showing the double-bellows ram, DAC, focusing cones, and other parts described in the text. (b) Schematic diagram of assembled probe, and expanded view of the focusing cone.

The two stages of the double-bellows ram are harnessed in the frame (1). In order to achieve additive force, the bottom plate (4) of the upper bellows does not make contact with the top plate (5) of the lower bellows. Instead, the two bottom plates (4 and 7) are connected together, as are the two top plates (2 and 5). The bellows are pressurized by passing gaseous or liquid ${ }^{4} \mathrm{He}$ into them through a stainless steel capillary tube (8). The overall ram diameter is only $26 \mathrm{~mm}$; its length is $76 \mathrm{~mm}$. It is capable of generating a maximum force of $5 \mathrm{kN}$ when pressurized 
to the freezing pressure of ${ }^{4} \mathrm{He}$ at $4.2 \mathrm{~K}$. The design can be scaled up easily by stacking more bellows stages in the same way. The force multiplies by the number of stages, e.g., 4 stages would give $10 \mathrm{kN}$. Hence, this design concept overcomes the size/force limitations of conventional single-bellows-driven DACs by stacking multiple bellows, geometrically in series (i.e., end to end), but mechanically in parallel so that the force from each bellows adds.

The DAC itself (18-25 in Fig. 1a) follows a modified Piermarini-Block design $[7,12]$. The DAC must be loaded and sealed before it is connected to the double-bellows ram. Initially, we employed methanol:ethanol (4:1 by volume) as the pressure medium in the DAC sample chamber, i.e., the gasket hole $(350 \mu \mathrm{m}$ in diameter). This medium, while usable, is non-hydrostatic for all pressures at $4.2 \mathrm{~K}$, and also has a broad FIR absorption band at $\approx 150 \mathrm{~cm}^{-1}$. Subsequently, we developed techniques for loading ${ }^{4} \mathrm{He}$ into the DAC [13]. Successful loading requires the $\mathrm{He}$ to be initially in the superfluid state. Solid ${ }^{4} \mathrm{He}$ is the most nearly hydrostatic of any possible DAC medium, and it does not suffer from having an absorption band in the FIR region of interest $\left(75-250 \mathrm{~cm}^{-1}\right)$.

In order to carry out FIR magnetospectroscopy, the DAC and double-bellows ram are assembled into the full probe system illustrated schematically in Fig. 1b. The DAC-ram apparatus is enclosed in a vacuum can $(E)$, and secured to a stainless steel tube that serves as the FIR light pipe $(F)$. There are also heating coils and thermistors (with low magnetic-field sensitivity) to vary and measure the temperature at the DAC in the range $2-300 \mathrm{~K}$. The vacuum can and light pipe can be evacuated or filled with He exchange gas, as required. During operation, this probe is inserted into the $36 \mathrm{~mm}$ bore of a standard superconducing magnet $(C)$, with the sample (inside the DAC) at the field-center. The FIR radiation is directed through the transmitting polyethylene window (vacuum seal) at the top port of the light pipe $(J)$, and focused onto the sample by the input paraboloidal cone $(D)$. The transmitted light is collected by the output paraboloidal cone, and projected to the Ga-doped Ge photoconductive detector (11 in Fig. 1a), placed far outside the magnet coil, yet still inside the $4.2 \mathrm{~K}$ region in order to operate within an acceptable noise level.

This apparatus offers the great advantage that FIR and visible-light experiments can be performed in close succession under the same conditions. The visible access is through the side port, quartz window, and sliding mirror ( $I$ in Fig. $1 \mathrm{~b}$ ) that can be moved in or out of the FIR light pipe as desired. This visible access allows us to measure photoluminescence (PL) from the sample or the ruby chip (for in situ pressure calibration). An example of the capabilities of this apparatus for visible magneto-PL is offered in Fig. 2. This shows PL spectra observed at $25 \mathrm{kbar}, 15 \mathrm{~K}$, and fields of $0-9 \mathrm{~T}$ in a Si-doped GaAs/AlGaAs multiple quantum well (MQW) structure (sample 1 in Fig. 3). The doublet arises from recombination of neutral $(X)$ and donor-bound $\left(D^{0} X\right)$ excitons; the quartet is due to the $R_{1}$ line of ruby, which exhibits Zeeman splitting according to its $T_{2}$ ground state. In fact, by calibrating this splitting, ruby can serve as a handy in situ magnetometer, in addition to its usual role as the barometer in DAC experiments [13].

The small FIR signals obtained for a sample in the DAC pose a formidable problem for Fourier transform magnetospectroscopy using conventional sources. 


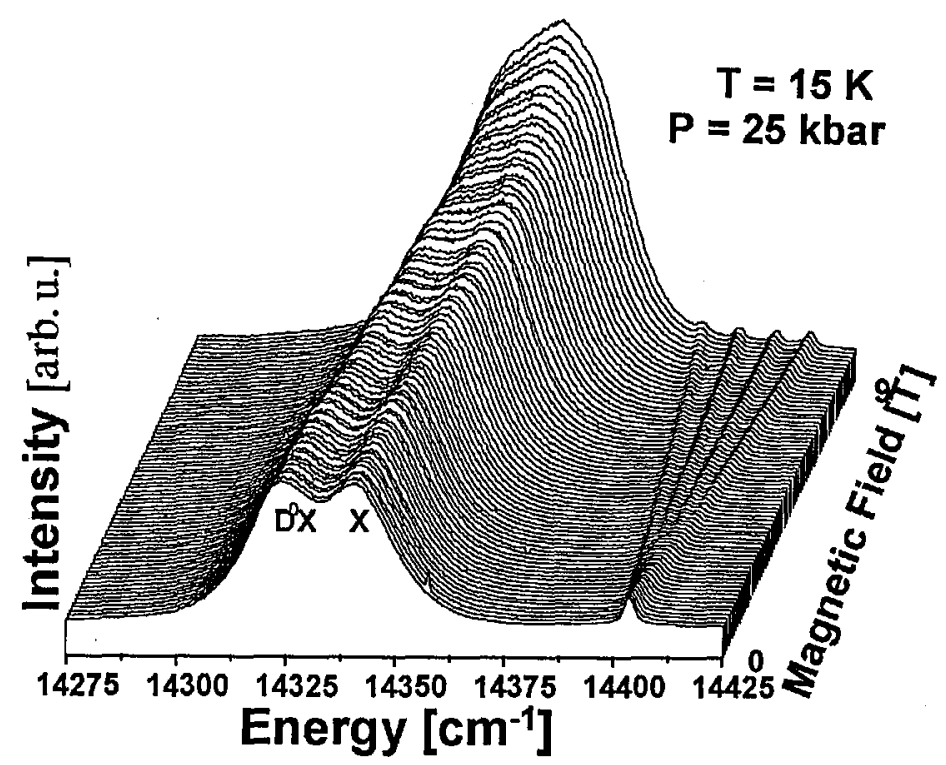

Fig. 2. High-pressure magneto-PL spectra of sample 1 recorded using the FIR-DAC apparatus.

We have found that wavelengths greater than $\approx 1 / 3$ the gasket hole diameter should be avoided because of diffraction effects. The gasket hole will still allow residual throughput for longer wavelengths. However, adhering to this rule-of-thumb helps to avoid spurious signals, as does the procedure of normalizing each magnetotransmission spectrum that exhibits resonant-absorption features by a background spectrum recorded at an off-resonance field. In order to optimize the optical system that directs the FIR light into and out of the DAC, we have employed special paraboloidal focusing and collecting cones (26 and 15 in Fig. 1a). Their shape (see Fig. 1b) has been designed to concentrate the FIR light at a spot $2.5 \mathrm{~mm}$ beyond the cone opening, i.e., at the sample position inside the DAC. This has allowed us to realize a factor $f \approx 2.3$ increase in throughput $[4,6]$ over standard focusing cones [14], which, by concentrating the light at the cone opening, increase losses in the front diamond anvil.

The design features of the FIR-DAC apparatus discussed in this section have enabled the Buffalo group to explore the effects of high pressure on a number of fascinating small energy excitations, several of which have only recently yielded to experimental investigations at ambient pressure. (See, for example, Refs. [15-18] and citations therein.) Here, we shall illustrate the potential of FIR-DAC experiments by discussing our latest results on cyclotron resonance (CR), neutral donors $\left(D^{0}\right)$, and negatively charged donors $\left(D^{-}\right)$in modulation-doped GaAs/AlGaAs MQWs. In earlier pressure-FIR studies of the $\mathrm{CR}$ and $D^{0}$ transitions in bulk GaAs, we explored the $D X$-center [19], and determined the effective mass of electrons up to the $\Gamma-X$ crossover at $40 \mathrm{kbar}[20]$ - a factor of two extension over existing results [21]. 
3. FIR magnetospectroscopy of CR, $D^{0}$, and $D^{-}$: samples and behavior at 1 atm

Four different $\mathrm{GaAs} / \mathrm{Al}_{0.3} \mathrm{Ga}_{0.7} \mathrm{As}$ multiple quantum well (MQW) samples, grown on [001] semi-insulating GaAs substrates by molecular beam epitaxy, are studied in this work. The samples have the same structure, as shown in Fig. 3. They are $\delta$-doped with $\mathrm{Si}$ donors in the center of the wells, and in the center of the barriers; the doping densities are tabulated in Fig. 3. For 1 atm and low pressures, at which the lowest energy of the conduction band edge (CBE) is at the QW $\Gamma$-point, essentially all of the barrier-donors release their electrons into the QWs. For the temperatures of our experiments $(4.2-30 \mathrm{~K})$, these excess electrons either reside in QW conduction states, where they can give rise to $\mathrm{CR}$, or they bind to the QW's donors contributing to the populations of $D^{0}$ and $D^{-}$. The wide $\mathrm{Al}_{0.3} \mathrm{Ga}_{0.7}$ As barriers prevent conduction electrons in the $\mathrm{QWs}$ from binding remotely to ionized barrier-donors. Also, the large average distance between $\mathrm{Si}$ atoms in the QWs, $\approx 700 \AA$, means that the QW donors are basically isolated. In prior work at ambient pressure, these samples have been fully characterized, and their FIR maganetotransmission spectra studied in detail $[16,22]$.

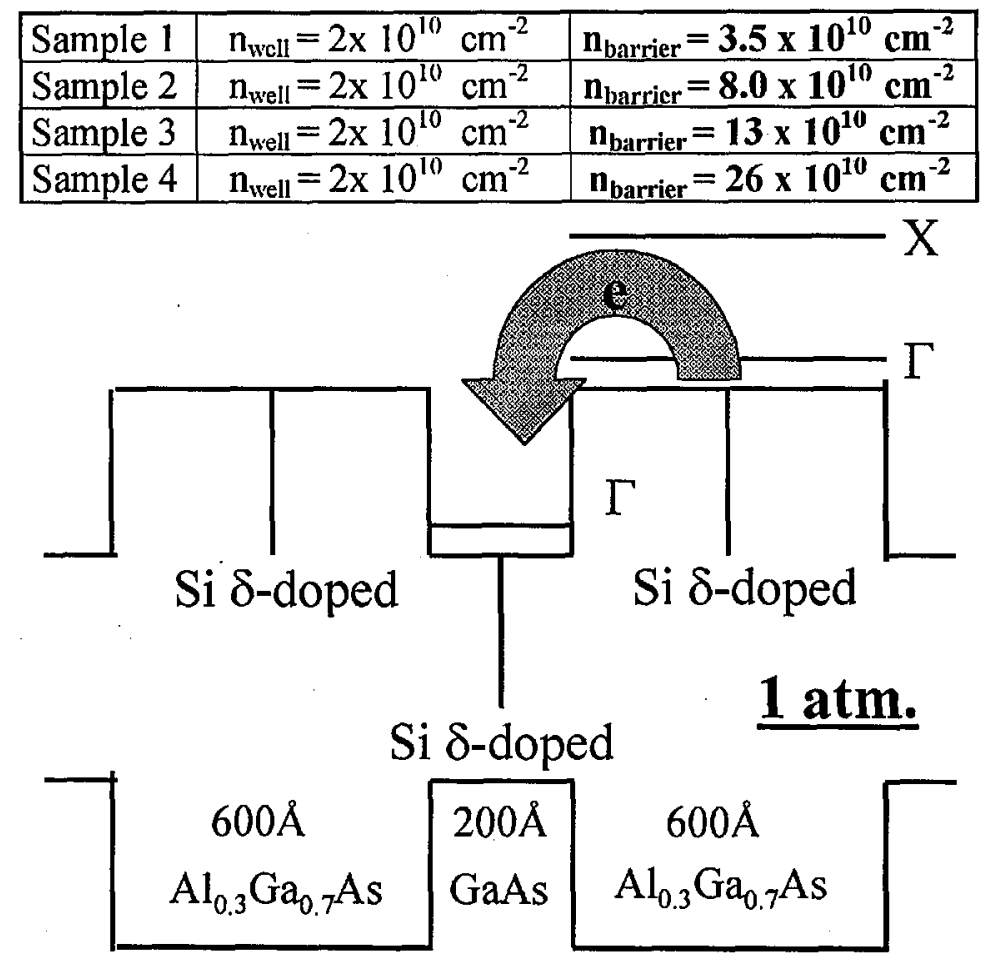

Fig. 3. Multiple quantum well samples studied in this work. Each sample had 20 repetitions of the structure shown.

For each sample, the Si donor density in the QWs is $2 \times 10^{10} \mathrm{~cm}^{-2}$. Since, in $\mathrm{GaAs}$, the ground state energy of $D^{-}$is found to be $\approx 0.48-0.85 \mathrm{Ry}^{*} \approx$ 
2.8-5.0 meV below that of $D^{0}(B=0$ and $2 \mathrm{D}$ limits) $[16,23]$, we expect that even sample 1 has sufficient barrier doping for $D^{-}$to dominate over $D^{0}$ at $1 \mathrm{~atm}$ and $4.2 \mathrm{~K}$. This is evident in Fig. 4 [22], which shows magnetotransmission spectra at $1 \mathrm{~atm}$ [given as $I(B=9 \mathrm{~T}) / I$ (off-resonance $B$ ) in units of \%-transmittance] for the samples tabulated in Fig. 3 plus a similar sample with lower doping (top trace). The progression with increasing total electron density (labels to the right) clearly shows how the $D^{0} 1 s \rightarrow 2 p^{+}$transition becomes dominated by the $D^{-}$singlet line when more than one electron per QW-donor is available. When more than two electrons per QW-donor is available, the $D^{-}$singlet line undergoes a blue shift that arises from many-electron effects [17]. The character of this transition then changes from that of an isolated $D^{-}$ion to that of a donor-bound magnetoplasmon. The increase in the CR-absorption strength with electron density is also seen in Fig. 4. Note that, for intermediate to high doping, the CR-line shape becomes strongly over absorbed, and we have found that the resulting line width can be used as a measure of the QW electron density [24].

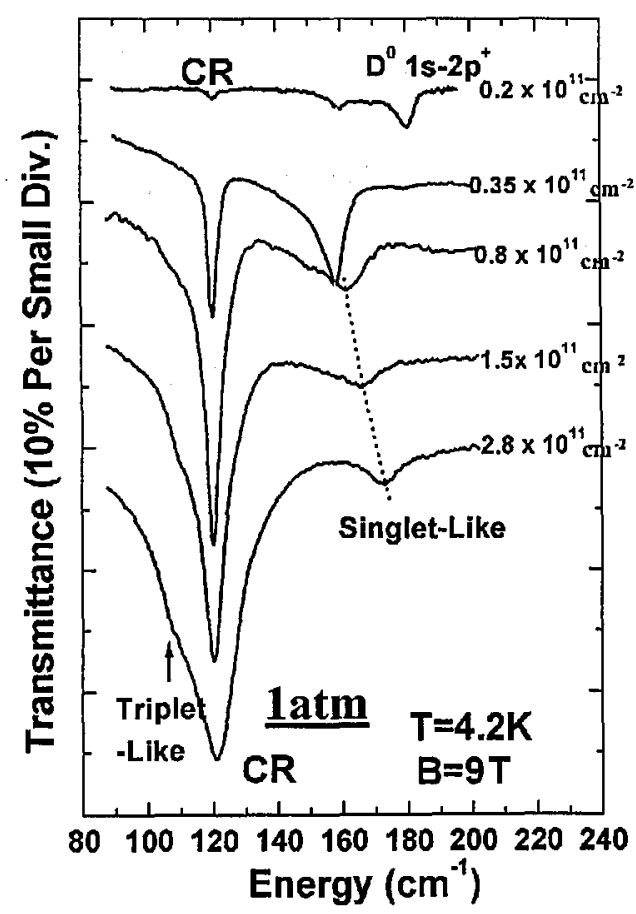

Fig. 4. FIR Fourier transform magnetotransmission spectra at ambient pressure for the present samples, and a similar sample with lower doping (top trace). After Ref. [22].

It is helpful to think of the isolated $D^{-}$singlet transition as the analog of the $D^{0} 1 s \rightarrow 2 p^{+}$transition, in which the second electron remains in the ground state. Of course, in reality, the energies of the $D^{-}$singlet ground and excited states are both modified by the Coulomb exchange terms due to repulsion between the two 
electrons [25]. Likewise, more complicated many electron effects mediate the evolution of the isolated $D^{-}$singlet line into the $D^{-}$singlet-like bound magnetoplasmon transition $[26,27]$.

\section{Effects of high pressure on the FIR magnetotransmission spectra of CR, $D^{0}$, and $D^{-}$}

Typical Fourier transform FIR magnetotransmission data for sample 1 at pressures of 22, 25, and 28 kbar are displayed in Figs. 5a-c, respectively. Each trace required 2-4 h integration time. At $22 \mathrm{kbar}$, and for all pressures $P \leq 22 \mathrm{kbar}$, the observed spectra are dominated by the CR and $D^{-}$singlet transitions. As expected, for a given $B$-field, these absorption lines are found to shift down in energy with increasing pressure. This also applies for the $D^{0} 1 s \rightarrow 2 p^{+}$transition, when present (see below). The downward energy shift is due to the increase in the conduction band effective mass with pressure, which decreases the slope of the field dependence for each transition. Figure 6 summarizes our results for the effects of pressure on the field dependencies of the three transitions observed in sample 1 .
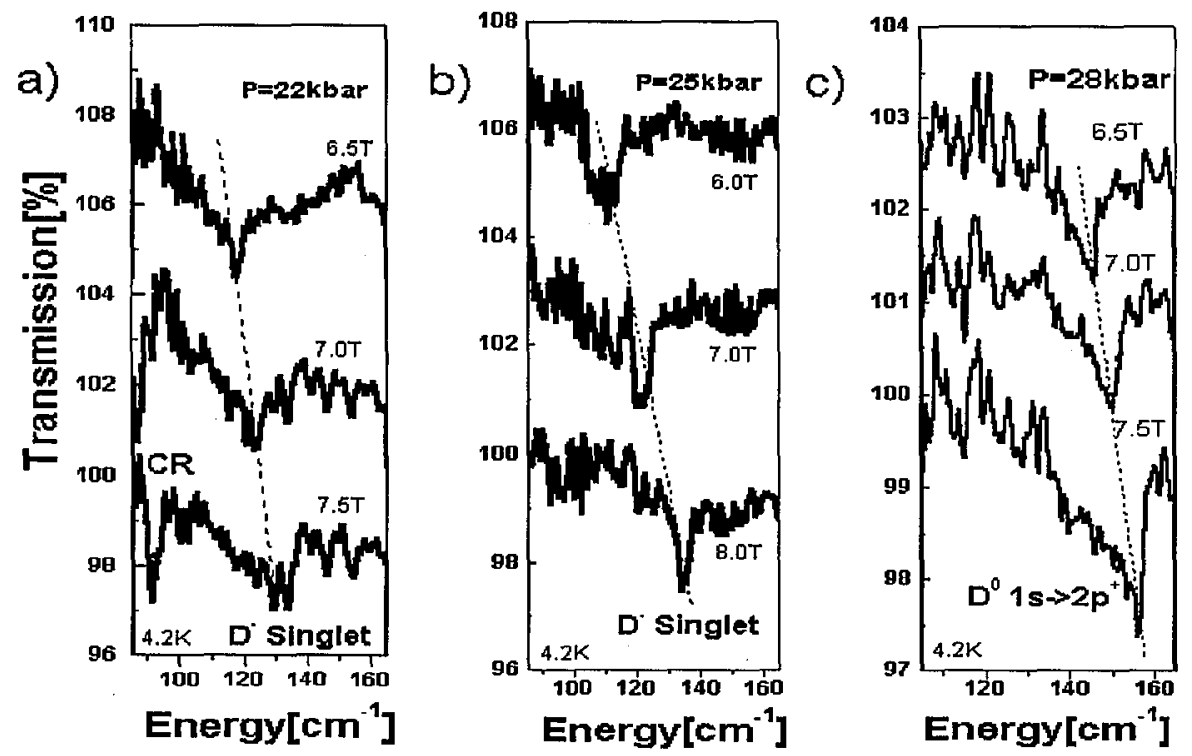

Fig. 5. FIR Fourier transform magnetotransmission spectra for sample 1 at (a) 22 kbar, (b) $25 \mathrm{kbar}$, and (c) $28 \mathrm{kbar}$.

The presence of CR in Fig. 5a shows that $n_{\mathrm{QW}}^{\mathrm{e}}$ (the total density of conduction band electrons in a $\mathrm{QW}$ ) has not been appreciably reduced in sample 1 for pressures up to $22 \mathrm{kbar}$. However, a significant change occurs on increasing to $25 \mathrm{kbar}$ (Fig. 5b). The CR line is no longer observed, but the $D^{-}$singlet transition is still present. This indicates that the pressure-induced transfer of electrons to barrier donors has commenced, and, as a result, $n_{\mathrm{QW}}^{\mathrm{e}}$ is now close to 
two electrons per $\mathrm{QW}$ donor (i.e., $\approx 4.0 \times 10^{10} \mathrm{~cm}^{-2}$ ). With further increase in pressure to $28 \mathrm{kbar}$ (Fig. 5c), both the $\mathrm{CR}$ and the $D^{-}$singlet transitions are no longer observed. Instead, the $D^{0} 1 s \rightarrow 2 p^{+}$absorption line appears, signalling that $n_{\mathrm{QW}}^{\mathrm{e}}$ has been reduced to one electron per donor. At pressures above $30 \mathrm{kbar}$, no absorption lines can be observed, signalling that the pressure-induced $\Gamma$ (well) $-X$ (barrier) crossover is complete. All of the spectral features were recovered on lowering the pressure.

Although the observed effects of pressure on $n_{\mathrm{QW}}^{\mathrm{e}}$ in sample 1 conform qualitatively to our expectations based on the $\Gamma$ (well) $-X$ (barrier) crossover, the drop off in $n_{\mathrm{QW}}^{\mathrm{e}}$ actually occurs somewhat below the threshold pressure predicted by theory. This deviation will be discussed further, after presenting the data for the other samples. We now wish to examine more closely the changes in field-dependence seen in Fig. 6.

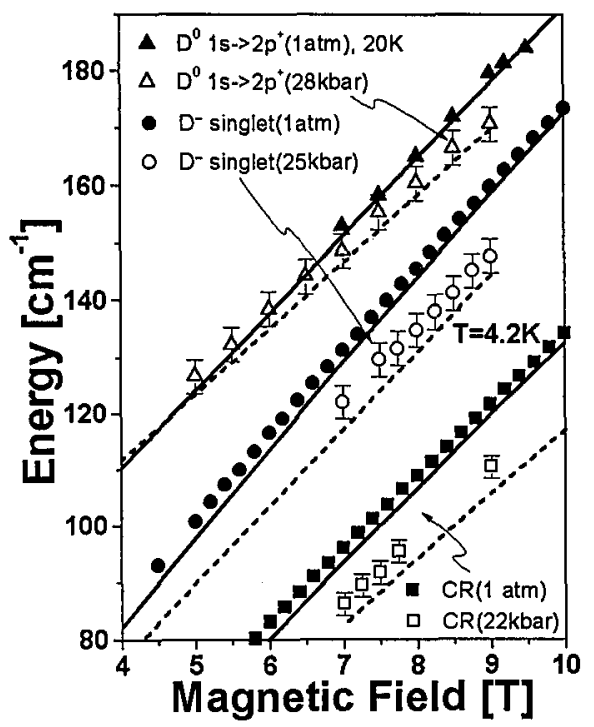

Fig. 6. Effects of applied pressure on the magnetic field dependence of the $\mathrm{CR}, D^{-}$ singlet, and $D^{\circ} 1 s \rightarrow 2 p^{+}$transitions observed in sample 1 .

The solid (1 atm) and dashed (elevated pressure) curves in Fig. 6 give theoretical results for the $D^{0} 1 s \rightarrow 2 p^{+}, D^{-}$singlet, and CR transitions computed for sample 1 using a treatment developed by Dzyubenko and Sivachenko [28]. In this treatment, the pertinent neutral donor, charged donor (singlet), and Landau-like states are determined by numerical diagonalization of the appropriate one- or two-electron Hamiltonians for a positive charge at the QW center. A basis of $\approx 3000$ Landau-oscillator and size-quantization wave functions is employed for this purpose. The calculations reported in Fig. 6 are performed for arbitrary magnetic fields $\geq 4 \mathrm{~T}$, and include the known pressure-dependencies of the effective mass and the dielectric constant in bulk GaAs [20,21]. Also incorporated 
(via a $k \cdot p$ treatment) are the effects of conduction band non-parabolicity, taken to be pressure-independent and equal to that in bulk GaAs at 1 atm. We compare, in Fig. 6, the measured and calculated field-variations at $1 \mathrm{~atm}$ and at the highest pressure for which each type of feature could be observed - CR (22 kbar), $D^{-}$singlet transition (25 kbar), and $D^{0} 1 s \rightarrow 2 p^{+}$transition (28 kbar). There is very good agreement between theory and experiment, although the calculated transition energies are in all cases slightly smaller than the measured ones. This discrepancy could arise from the theory using too small a basis set, and/or too large a non-parabolicity correction.

The calculations for the $D^{-}$singlet transition are performed for an isolated $D^{-}$ion, and therefore exclude the many electron effects which tend to blue shift the $D^{-}$singlet-like bound magnetoplasmon resonance. In order to better determine this blue shift at high pressure, we have fit the numerical results for the calculated isolated- $D^{-}$singlet transition. The best fit was

$E_{D_{\text {snglt }}}^{\text {calc }}(B, P)=23 \mathrm{~cm}^{-1}+15 \frac{\mathrm{cm}^{-1}}{\mathrm{~T}} B+0.018 \frac{\mathrm{cm}^{-1}}{\mathrm{kbar}} P-0.065 \frac{\mathrm{cm}}{\mathrm{T} \cdot \mathrm{kbar}} B \cdot P$

valid for $4 \mathrm{~T} \leq B \leq 9 \mathrm{~T}$ and $P \leq 30$ kbar within a precision set to match our experimental accuracy.

Notice, in Fig. 6, that the $1 \mathrm{~atm}$ and elevated-pressure field-variations appear to cross each other at magnetic fields that are different for the $D^{0} 1 s \rightarrow 2 p^{+}$ transition and the $D^{-}$singlet transition. Both the theoretical and the experimental results indicate that, for a given elevated pressure, this crossing should occur at a higher field for the $D^{0} 1 s \rightarrow 2 p^{+}$case (at $\approx 5 \mathrm{~T}$ in Fig. 6) than for the $D^{-}$singlet case (at a field below our measurement range). Such behavior can be understood by considering these transitions in the high- and low-field limits for a strictly 2D situation, as depicted in Fig. 7. Then, it is straightforward to show (see Ref. [25]) that the $D^{-}$singlet and the $D^{0} 1 s \rightarrow 2 p^{+}$transitions vary with $B$-field asymptotically to the same high-field energy position. This energy is $E_{\mathrm{D}^{0}} / 2$ above the CR energy, where $E_{\mathrm{D}^{0}}=\sqrt{\pi / 2} e^{2} / \varepsilon \ell$ with $\ell$ the magnetic localization length. This means that, at constant high $B$, applied pressure causes both transitions to move to lower energy at essentially the same rate as the CR. On the other hand, in the low field limit, the $D^{-}$singlet and $D^{0} 1 s \rightarrow 2 p^{+}$transitions increase in energy with pressure at different rates - set by the change in separation between their respective zero-field hydrogenic levels. These rates should be $3.55 \times \mathrm{d} R_{y}^{*} / \mathrm{d} P$ for the $D^{0} 1 s \rightarrow 2 p$ transition and $\approx 0.5 \times \mathrm{d} R_{y}^{*} / \mathrm{d} P$ for the $D^{-}$singlet transition $[23,29]$. The difference in prefactors explains the difference in crossing fields, since $\mathrm{d} R_{y}^{*} / \mathrm{d} P$ (being determined by the pressure variations of $m_{\mathrm{e}}^{*}$ and $\varepsilon$ ) is the same for both types of transitions. Our experimental data correspond to the intermediate magnetic field regime indicated by the solid square in Fig. 7. Overall, this physical picture is in qualitative agreement with the experimental results in Fig. 6.

High-pressure FIR magnetotransmission measurements were performed on the other samples listed in Fig. 3 using both Fourier transform spectroscopy and laser magnetospectroscopy (i.e., scanned $B$-field and fixed $\lambda=118.8 \mu \mathrm{m}$, primarily for sample 2). The Fourier transform results for sample 3 , which has an intermediate level of doping, are shown in Fig. 8. At $18 \mathrm{kbar}$ (Fig. 8a) the spectra for this sample are characterized by a strong $\mathrm{CR}$ line, and a weaker absorption feature at 


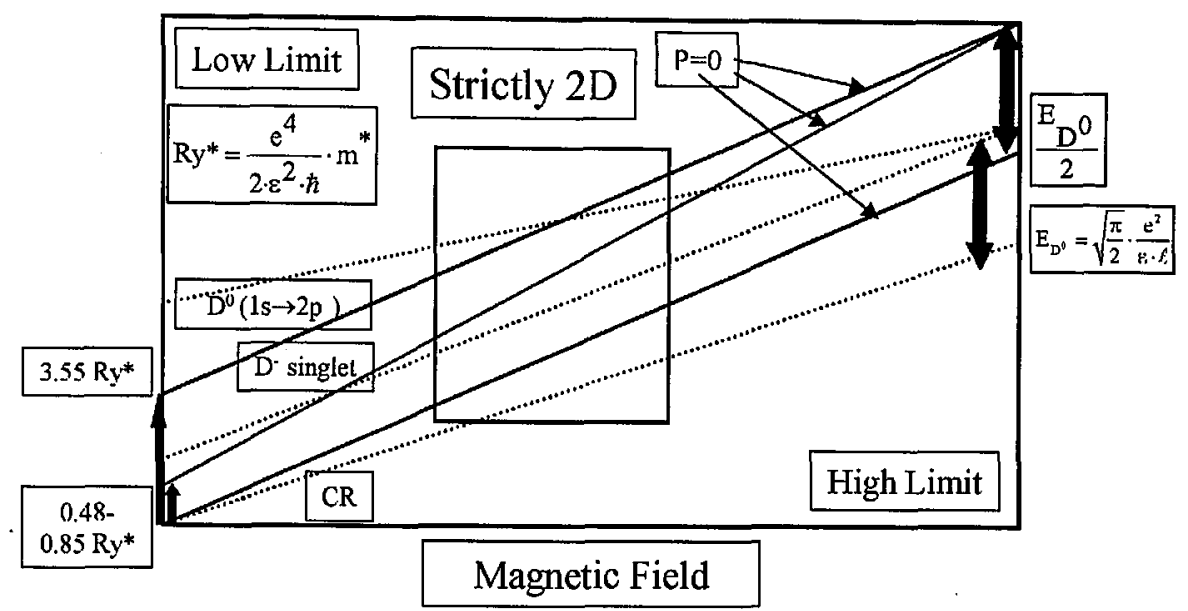

Fig. 7. Representation of the effects of pressure on the $D^{0} 1 s \rightarrow 2 p^{+}, D^{-}$singlet, and CR transitions in the low-field and the high-field limits for a strictly $2 \mathrm{D}$ system. Note the different crossing points of the $1 \mathrm{~atm}$ and high-pressure curves for each type of transition.
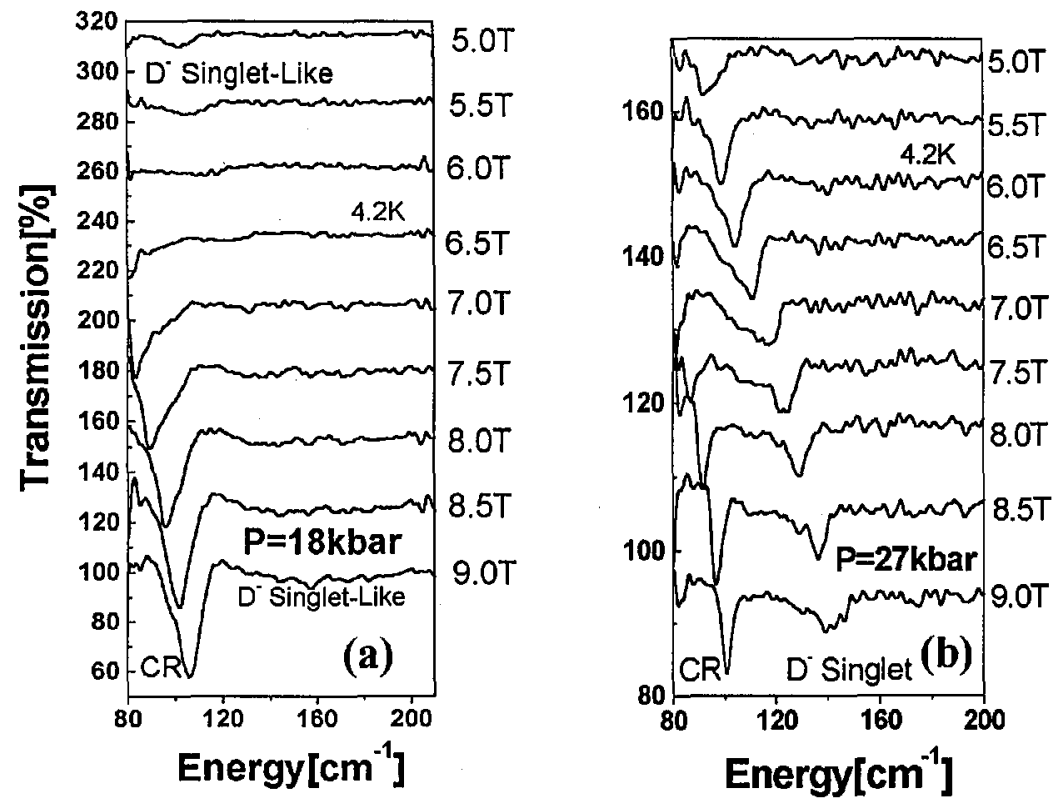

Fig. 8. FIR Fourier transform magnetotransmission spectra for sample 3 at (a) $18 \mathrm{kbar}$, and (b) 27 kbar.

higher energy. The latter is identified as the $D^{-}$singlet-like transition, based on its blue-shifted energy position relative to the analogous singlet transition for an isolated $D^{-}$ion [17], given by Eq. (1). We observe also that, with increasing field in the range 5-9 T, the $D^{-}$peak tends to weaken and become broader, an intriguing 
field behavior that was observed in this sample for all pressures below $20 \mathrm{kbar}$. At 27 kbar (Fig. 8b) the line width of the CR transition has become substantially narrower than at lower pressure, and its intensity has decreased sharply relative to the $D^{-}$feature. The label $D^{-}$singlet is now used for the latter feature, since it is no longer blue-shifted above the energy given by Eq. (1). Furthermore, the shape and intensity of the $D^{-}$singlet peak are no longer affected strongly by the magnetic field, as they were for $P \leq 20 \mathrm{kbar}$.

The observed decreases in the width and strength of the CR line, and the loss of the blue shift for the $D^{-}$feature, reflect the drop in $n_{\mathrm{QW}}^{\mathrm{e}}$ caused by the pressure-induced $\Gamma$ (well) $X$ (barrier) crossover. As mentioned, the narrowing of the CR peak is due to reduced over-absorption [24]. Similar behavior was seen for sample 4. In fact, using the 1 atm magnetotransmission results for our samples [22], we have established an approximate linear relationship between the $C R$ line width and the electron density, which then was employed to determine $n_{\mathrm{QW}}^{\mathrm{e}}$ as a function of pressure.

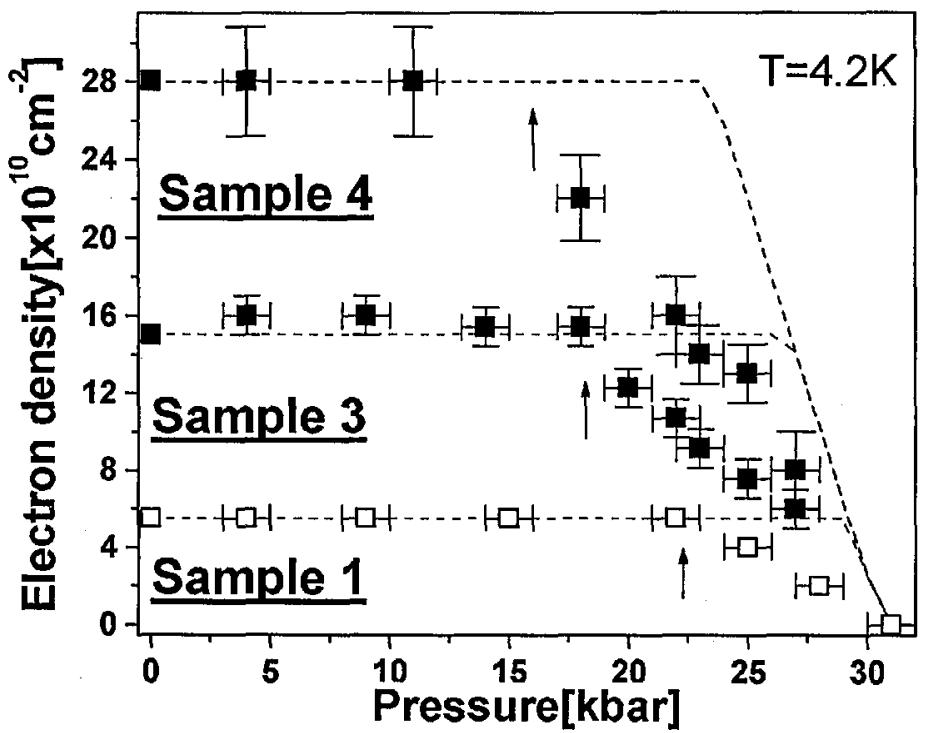

Fig. 9. Pressure dependence of $n_{Q W}^{\mathbf{e}}$ in samples 1,3 , and 4 determined from the present measurements (data points) and calculated via the model solid approach (dashed curves).

Figure 9 presents a summary of our final findings for the pressure-tuning of the electron density. The dashed curves in this figure are theoretical results for $n_{\mathrm{QW}}^{\mathrm{e}}$ vs. pressure in our explicit samples. They are computed using the model solid theory of Van de Walle to describe the band offsets [30], combined with a variational solution of Schrödinger's equation for the QW states, and a self-consistent treatment of the potential via Poisson's equation to properly account for the changes 
in band bending as the pressure causes charge to be transferred between the wells and the barriers [29,31]. The input parameters for this calculation - band gaps, deformation potentials, pressure-dependent effective masses, elastic constants, and bowing constants - are all taken from known experimental values in the well characterized GaAlAs system [30]. Hence, our confidence level is high for the theoretical curves. Overall, in Fig. 9, experiment and theory agree: $n_{\mathrm{QW}}^{\mathrm{e}}$ remains unaffected up to a threshold pressure that is lower for higher doping, above which $n_{\mathrm{QW}}^{\mathrm{e}}$ decreases linearly until complete depletion at $31 \mathrm{kbar}$ due to the $\Gamma$ (well) $-X$ (barrier) crossover. However, there is a systematic discrepancy for these threshold pressures, which seem to be $\approx 7 \mathrm{kbar}$ lower than predicted theoretically.

This difference indicates the presence of another trapping level located $\approx 70 \mathrm{meV}$ below the Si-donor level associated with the $X$-point in the barriers. This trap cannot be the DX-center, nor any other metastable trap. This is because such centers should be completely and persistently photoionized during our experiments, due to the frequent ruby pressure calibrations involving exposure, at $4.2 \mathrm{~K}$, to $300 \mathrm{~mW}$ of $514 \mathrm{~nm}$ light. A likely origin for these barrier traps is nominal levels of Se donors (on As sites). Se is a known residual impurity in MBE grown GaAlAs, and there is evidence that its $X$-associated state is non-metastable and has a binding energy of $\approx 100 \mathrm{meV}$ (or about $60 \mathrm{meV}$ below the $X$-level of Si donors in the GaAlAs system) [3, 32]. Thus, the low fall-off pressures for $n_{\mathrm{QW}}^{\mathrm{e}}$ (Fig. 9) could be accounted for by a few $\times 10^{10} \mathrm{~cm}^{-2}$ Se donors in the barriers of our samples.

The high pressure results for sample 4 (with the highest barrier-doping, $26 \times 10^{10} \mathrm{~cm}^{-2}$ ), reveal interesting new behavior that was not anticipated from the previous 1 atm work. Fourier transform FIR transmission spectra for this sample at $27 \mathrm{kbar}$ are shown in Fig. 10a (for 5.5-7.5 T) and Fig. 10b (for 7.5-9.0 T). The results for fields up to $\approx 7.5 \mathrm{~T}$ exhibit the normal behavior. At low pressures, one observes strong $\mathrm{CR}$ and $D^{-}$singlet-like peaks. With increasing pressure, the intensity (and width) of the $\mathrm{CR}$ and the blue shift of the $D^{-}$singlet-like peak decrease, as the $\mathrm{QW}$-electrons are lost to Se donors (above $\approx 18 \mathrm{kbar}$ ) and $\mathrm{Si}$ donors (above $\approx 23 \mathrm{kbar}$ ). Again, for $P \geq 31 \mathrm{kbar}$, no FIR absorption peaks can be seen. On the other hand, for fields $B \geq 7.5 \mathrm{~T}$, we find that the $D^{-}$singlet-like peak weakens and broadens, and a new peak is observed at $\approx 160-180 \mathrm{~cm}^{-1}$ (Fig. 10b). This has no parallel in the 1 atm results for any samples studied so far. Although the new high-energy peak is most pronounced at $27 \mathrm{kbar}$ (where the spectra show a clear doublet), it first appears weakly at $\approx 6 \mathrm{kbar}$, and, thereafter, its strength and energy increase with pressure relative to the $D^{-}$singlet-like peak. No anomalies are seen for the $\mathrm{CR}$ line in the 7.5 $\mathrm{T}$ region at any pressure.

A summary of the field dependence at different pressures of the transition energies observed in sample 4 appears in Fig. 11. The calculated results discussed earlier for the isolated $D^{-}$singlet transition (viz., Eq. (1)) and the $D^{0}$ $1 s \rightarrow 2 p^{+}$transition are again represented by solid (1 atm) and dashed (high-pressure) curves. For $B \leq 7.5 \mathrm{~T}$, the energy difference between the theoretical $D^{-}$curves and the corresponding data points gives the blue shift of the $D^{-}$ singlet-like peak. This blue shift decreases with pressure in line with the decrease 

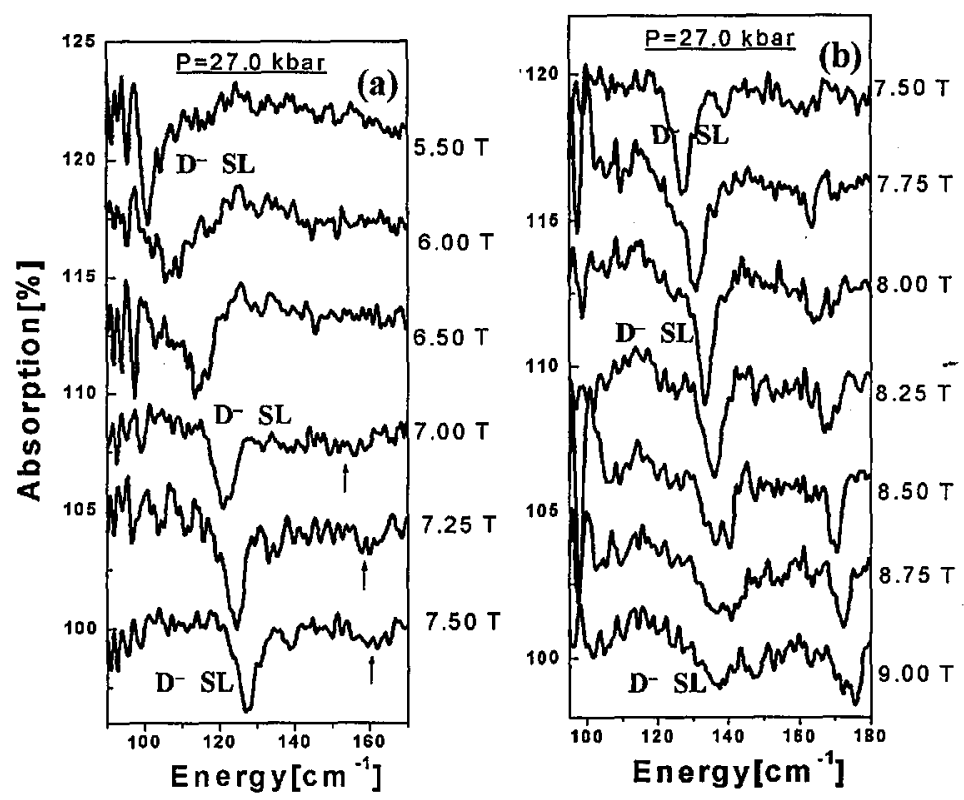

Fig. 10. FIR Fourier transform spectra for sample 4 at $27 \mathrm{kbar}$ for two ranges of magnetic field: (a) 5.5-7.5 T, and (b) 7.5-9.0 T. The arrow in (a) marks the probable position of the new high-energy peak.

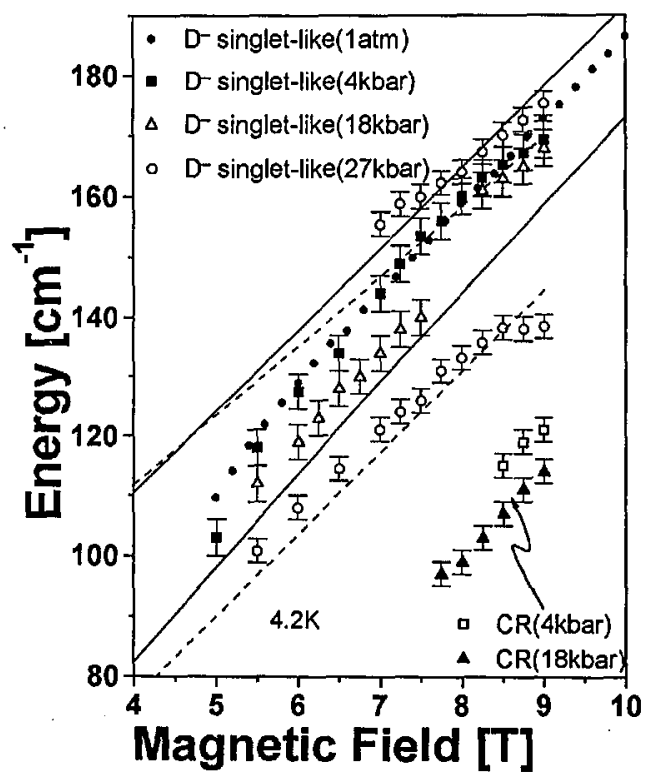

Fig. 11. Effects of applied pressure on the magnetic field dependence of the CR, $D^{-}$ singlet-like, and $D^{0} 1 s \rightarrow 2 p^{+}$transitions in sample 4 . Note the branching anomaly and apparent anticrossing for the $D^{-}$singlet-like transition. 
of $n_{\mathrm{QW}}^{\mathrm{e}}$ (Fig. 9). For $B \geq 7.5 \mathrm{~T}$, the splitting between the $D^{-}$singlet-like peak and the new high-energy peak is apparent in the $18 \mathrm{kbar}$ and $27 \mathrm{kbar}$ data. In fact, Fig. 11 suggests that the field dependence of the $D^{-}$singlet-like transition breaks into two branches that are subject to an anticrossing interaction. Also, with increasing pressure, the energy of the upper branch seems to evolve (see $27 \mathrm{kbar}$ data in Fig. 11) into the energy range expected for the $D^{0} 1 s \rightarrow 2 p^{+}$transition.

It is interesting to speculate on the origin of this branching effect. One possible cause is misalignment of the sample in the DAC relative to the $B$-field. Then, the $B$-component in the QW plane can couple impurity and Landau states associated with the first and second QW subbands. This can be ruled out because the subband separation $\left(\approx 590 \mathrm{~cm}^{-1}\right)$ is far from the observed frequencies $\left(\approx 120-180 \mathrm{~cm}^{-1}\right)$, and the branch splitting $\left(\approx 25 \mathrm{~cm}^{-1}\right)$ is unreasonably large for the estimated maximum tilt angle $\left(\approx 15^{\circ}\right)$. A more realistic possibility is that the branching arises from coupling to a resonant localized level. If so, one needs to explain the peculiar pressure and field dependencies of this coupling - namely, that it increases in strength with pressure, but leads to a splitting at $\approx 7.5 \mathrm{~T}$ independent of pressure. On this basis, for example, we probably can exclude unintentional impurities in the $Q W s$ (e.g., Se) as the source of the branching. Alternatively, instead of an anticrossing mechanism, the branching might be due to spatial inhomogeneities in the QWs such that the $D^{-}$and $D^{0}$ species exist concurrently in different regions. The required inhomogeneities could be caused by the Coulomb fluctuations from, e.g., charged interface defects or nominal levels of barrier impurities. Again, for this mechanism, the pressure and field dependencies must be carefully considered.

\section{Summary}

A DAC apparatus for performing high-pressure FIR magnetospectroscopy has been described in this review. The importance of being able to tune the pressure in situ has been emphasized. We describe experiments carried out with this apparatus on GaAs/AlGaAs multiple quantum wells that are modulation doped with $\mathrm{Si}$ in both the wells and the barriers. With increasing pressure, there is a progression of the observed QW spectra from being dominated by CR and the $D^{-}$ singlet (or singlet-like) peaks, to showing the $D^{0} 1 s \rightarrow 2 p^{+}$transition. The reason is the transfer of electrons from the QWs back to their parent $\mathrm{Si}$ donors in the barriers due to the pressure-induced $\Gamma$ (well) $-X$ (barrier) crossover. The threshold pressures for the fall off of $n_{\mathrm{QW}}^{\mathrm{e}}$ in our samples are systematically lower than predicted by model solid calculations. The most likely cause is the presence in the barriers of nominal Se impurities with a level $\approx 100 \mathrm{meV}$ below the $X$-point. For low and intermediate doping, the effects of pressure on the field dependence of the $\mathrm{CR}, D^{0} 1 s \rightarrow 2 p^{+}$, and $D^{-}$singlet transitions are primarily determined by the pressure dependencies of the electron effective mass and the dielectric constant. Detailed numerical calculations that include these effects agree very well with the experimental results. However, for high doping, a new transition at energies above the $D^{-}$singlet-like peak is observed for $P \geq 6 \mathrm{kbar}$ and $B \geq 7.5 \mathrm{~T}$. This leads to an apparent branching in the field dependence of the $D^{-}$singlet-like transition, an intriguing behavior which merits further experimental and theoretical study. 


\section{Acknowledgments}

The authors are grateful to Dr. W. Schaff of Cornell University for growth of the excellent samples. This work was supported by NSF grant \# DMR-9624029 and ONR contract \# N0001489J1797.

\section{References}

[1] See for example, W. Paul, Solids Under Pressure, McGraw Hill, New York 1963, Ch. 8.

[2] W.A. Challener, J.D. Thompson, Appl. Spectrosc. 40, 298 (1986).

[3] J.E. Dmochowski, P.D. Wang, R.A. Stradling, Semicond. Sci. Technol. 6, 118 (1991).

[4] S.N. Holmes, M.M. Li, B.A. Weinstein, B.D. McCombe, Jpn. J. Appl. Phys. 32, Suppl. 32-1, 340 (1993).

[5] J.A. Wolk, M.B. Kruger, J.N. Heyman, W. Walukiewicz, R. Jeanloz, E.E. Haller, Phys. Rev. Lett. 66, 774 (1991).

[6] R.J. Chen, B.A. Weinstein, Rev. Sci. Instrum. 67, 2883 (1996).

[7] B.A. Weinstein, in: High Pressure Science and Technology, MRS Proceedings, Eds. C. Homan, R.K. MacCrone, E. Whalley, Vol. 22, Part III, North-Holland, New York 1984, p. 341.

[8] I.F. Silvera, R.J. Wijngaarden, Rev. Sci. Instrum. 56, 121 (1985).

[9] W.B. Daniels, M.G. Ryschkewitsch, Rev. Sci. Instrum. 54, 115 (1983).

[10] T. Kobayashi, Rev. Sci. Instrum. 56, 255 (1985).

[11] United States Patent \#5,693,345, Dec. 2, 1997.

[12] G.J. Piermarini, S. Block, Rev. Sci. Instrum. 46, 973 (1975).

[13] J.G. Tischler, Ph.D. thesis, unpublished, 2000.

[14] W.T. Welford, R. Winston, High Collection Nonimaging Optics, Academic, New York 1989.

[15] S. Huant, S.P. Najda, B. Etienne, Phys. Rev. Lett. 65, 1486 (1990).

[16] S. Holmes, J.-P. Cheng, B.D. McCombe, W. Schaff, Phys. Rev. Lett. 69, 2571 (1992).

[17] Z.X. Jiang, B.D. McCombe, P. Hawrylak, Phys. Rev. Lett. 81, 3499 (1998).

[18] B.D. McCombe, Z.X. Jiang, J.G. Tischler, B.A. Weinstein, P. Hawrylak, Acta Phys. Pol. A 96, 559 (1999).

[19] R.J. Chen, Z.X. Jiang, B.A. Weinstein, B.D. McCombe, in: Proc. 23 Intl. Conf.: The Physics of Semiconductors, Vol. 4, Ed. M. Scheffler, World Sci., Singapore 1996, p. 2753.

[20] Z.X. Jiang, R.J. Chen, J.G. Tischler, B.A. Weinstein, B.D. McCombe, Phys. Status Solidi $B$ 198, 41 (1996).

[21] Z. Wasilewski, R.A. Stradling, Semicond. Sci. Technol. 1, 264 (1986).

[22] J.-P. Cheng, Y.J. Wang, B.D. McCombe, W. Schaff, Phys. Rev. Lett. 70, 489 (1993).

[23] D.M. Larsen, S.Y. McCann, Phys. Rev. B 46, 3966 (1992).

[24] T.A. Kennedy, R. Wagner, B. McCombe, J.J. Quinn, Solid State Commun. 18, 275 (1976). 
[25] A.H. MacDonald, D.S. Ritchie, Phys. Rev. B 33, 8336 (1986); A.H. MacDonald, Solid State Commun. 84, 109 (1992).

[26] A.B. Dzyubenko, Yu.E. Lozovik, JETP 77, 617 (1993).

[27] P. Hawrylak, Phys. Rev. Lett. 72, 2943 (1994).

[28] A.B. Dzyubenko, A.Yu. Sivachenko, JETP Lett. 57, 507 (1993); Phys. Rev. B 48, 14690 (1993).

[29] G. Bastard, Wave Mechanics Applied to Semiconductor Heterostructures, Halsted Press, New York 1988, Ch. 2.

[30] C.G. Van de Walle, Phys. Rev. B 39, 1871 (1989).

[31] S.K. Singh, Ph.D. thesis, unpublished, 1998.

[32] C. Skierbiszewski, private communication. 